\title{
Modeling diffusion on heterogeneous lattices: Derivation of general analytical expressions and verification for a two-dimensional square lattice
}

\author{
A. Tarasenko, ${ }^{1,2, *}$ L. Jastrabik, ${ }^{1}$ and T. Müller ${ }^{3}$ \\ ${ }^{1}$ Institute of Physics, Academy of Sciences of the Czech Republic, Na Slovance 2, 18221 Prague 8, Czech Republic \\ ${ }^{2}$ Institute of Physics, National Academy of Sciences of Ukraine, Prospect Nauki 46, UA-03039 Kyjv 39, Ukraine \\ ${ }^{3}$ Central Institute for Applied Mathematics, Research Centre Jülich, D-52425 Jülich, Germany
}

(Received 31 October 2006; published 2 February 2007)

\begin{abstract}
In order to model diffusion for real crystals, it is necessary to acknowledge that for many chemically and physically interesting classes of compounds (e.g., semiconductors, ionic solids, alloys), there are several different binding sites of possibly widely different character. In contrast to the majority of existing lattice-gas models, which ignore this aspect by assuming equivalent lattice sites, we investigate the diffusion of particles on a heterogeneous lattice with two kinds of nonequivalent sites. General analytical expressions for the chemical and jump diffusion coefficients have been derived in the case of strong inhomogeneity for lattices of different symmetries and dimensionality. It is shown that the character of the particle migration depends crucially on the relative jump frequencies of particles sitting in deep and shallow sites. If these frequencies differ insignificantly, particle diffusion proceeds by single uncorrelated jumps. In the opposite case of widely differing jump frequencies, particles perform pairs of strongly correlated jumps. We have calculated density dependencies of the diffusion coefficients and some thermodynamic quantities for different temperatures and signs of the lateral pairwise interaction between the particles. The analytical data obtained by the real-space renormalization-group method have been compared with the numerical data obtained by Monte Carlo simulations. Almost perfect agreement between the respective results has been found.
\end{abstract}

DOI: 10.1103/PhysRevB.75.085401

PACS number(s): 64.60.Ak, 66.10.Cb, 64.60.Cn, 66.30.Dn

\section{INTRODUCTION}

The diffusive mass transfer controls the rates of a multitude of physical, chemical, and biological processes. Theory primarily aims at understanding the details of the migration process in these application areas. The theoretical description of various kinetic phenomena observed in experimental studies presents a considerable challenge. Appropriate models must reflect the elementary microscopic migration act of particles, which depends on the structure and mutual particleparticle interaction. Therefore, it is not surprising that a great deal of effort has been devoted to developing the simplest possible models, which offer the advantage of exact treatment, despite oversimplifying the real phenomena. To include all these aspects, it is most convenient to employ the lattice-gas models. In these models, particles perform stochastic jumps among the sites of a discrete lattice. During migration acts, affected by thermal activation, the particles have to surmount barriers separating the sites. The effective barrier height depends on the specific atomic environment and, as a consequence of the particle-particle interaction, also on the number and configuration of the neighbor particles. In the majority of models, the particles occupy the equivalent lattice sites formed by the minima of the potential relief. Such simplification differs substantially from the real crystals, where different binding sites have been experimentally identified.

In general, the determination of the diffusion coefficient requires the solution of a kinetic equation for a many-particle system. However, under simplifying assumptions such as slowly varying particle density inhomogeneities and neglecting memory effects, the problem can be reduced to the calculation of purely thermodynamic quantities: free energy and its derivatives over chemical potential and interaction parameters. ${ }^{1-3}$ This approach has been used widely for the theoretical treatment of particle diffusion. The task of computing thermodynamic quantities is substantially simpler and computationally less expensive by orders of magnitude than direct Monte Carlo (MC) simulations of kinetic phenomena, which suffer from the statistical uncertainty of the results. It turns out that the approximations involved when connecting diffusion coefficients with thermodynamical quantities are rather reliable for the lattice-gas systems. Results obtained by different analytical methods [quasichemical approximation, cluster variation approach, real-space renormalization group (RSRG) method, and others] show quite good agreement with the numerical data (kinetic MC simulations). The analytical expressions for the diffusion coefficients are quite simple and valid over wide regions of particle density and temperature down to the subcritical values. These expressions work perfectly in homogeneous lattices with different dimensions and symmetries. Yet there is no general upper bound to the errors established for these approximations just as there is no guarantee for MC results to converge to the required statistical accuracy for the general case with limited computer resources. Compared to purely numerical schemes, the theoretical methods of statistical mechanics reveal correlations between the kinetic coefficients and some thermodynamical quantities, thereby providing the basis for additional insight.

In the case of inhomogeneous lattices, the ordinary expressions for the diffusion coefficients give qualitatively correct results for high temperatures only, when the lattice inhomogeneity is small and really can be neglected. With decreasing temperature, the deviations between analytical results and MC data grow considerably. The diffusion on het- 
erogeneous lattices was studied theoretically in someprevious investigations. Diffusion in a lattice gas on an fcc(100) surface was investigated in Refs. 4 and 5 and on a square lattice without lateral interaction between the adsorbed particles in Ref. 6. It was shown that the surface inhomogeneity substantially changes the particle migration. The coverage dependencies for tracer, jump, and chemical diffusion coefficients differ qualitatively from those obtained for the homogeneous lattice.

In the present work, we have investigated the effect of the lattice inhomogeneity on the particle diffusion. The purpose of the investigation is to present the analytical expressions for the jump and chemical diffusion coefficients that describe the particle diffusion over the inhomogeneous lattices with two kinds of adsorption sites. We derive such simple expressions that occur quite universally and are valid for many types of inhomogeneous lattices. Using the RSRG approach and MC simulations, we check these expressions for a simple square lattice. We have obtained the density dependencies of the tracer, jump, and chemical diffusion coefficients for some representative temperatures. Also the mean square density fluctuations or isothermal susceptibility, the adsorption isotherms (the particle density as a function of the chemical potential), and the pair correlation function for the nearest-neighbor sites have been calculated in the whole density region for different temperatures. It has been shown that for significant lattice inhomogeneities at low temperatures, there is a rather specific behavior of the particle migration over the lattice. The ordinary analytical expressions fail even to qualitatively describe density dependencies of jump and chemical diffusion coefficients. The new expressions derived in this work fit almost perfectly the MC data obtained at low temperatures.

The outline of this paper is as follows. The model and derivation of the diffusion equation and the expression for the diffusion coefficient are described in Sec. I. The RSRG approach employed in this work is described in Sec. II. The MC technique is explained in Sec. III. Our results are presented and discussed in Sec. IV.

\section{DIFFUSION OF PARTICLES ON INHOMOGENEOUS LATTICES}

In the following, we consider an ideal regular lattice with coordination number $z$. There are deep $(d)$ and shallow $(s)$ sites, arranged in alternating order (an example of such a lattice of square symmetry is shown in Fig. 1). Hence, the lattice may be regarded as composed of two equivalent interleaved sublattices comprised of deep and shallow sites only. If the adsorption energies, $\varepsilon_{d}, \varepsilon_{s}$, for the $d$ and $s$ sites, respectively, are large relative to the thermal energy, $k_{B} T$, the particles will almost populate the minima, jumping occasionally to empty, nearest-neighbor sites. A set of occupation numbers, $\left\{n_{i}\right\}$, describes all possible states of the particle system,

$$
n_{i}= \begin{cases}1 & \text { if the } i \text { th site is occupied } \\ 0 & \text { if the } i \text { th site is empty }\end{cases}
$$

In thermodynamic equilibrium, the system behavior is described by the grand partition function,

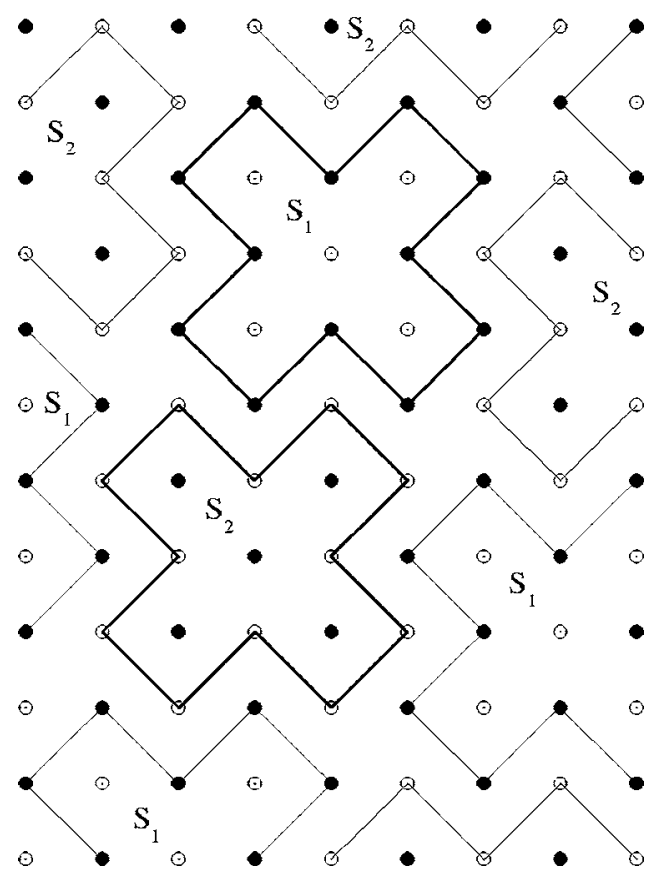

FIG. 1. Schematic view of a square lattice with two kinds of sites. The filled and open circles denote deep and shallow sites. The blocks of the $17 \times 2$ RSRG transformation are shown by solid lines.

$$
Q=\sum_{\left\{n_{i}\right\}} \exp \left(\mu N_{a}-H_{a}\right)
$$

or its corresponding potential, $F=N^{-1} \ln Q$, termed free energy. Here $\mu, N_{a}$, and $H_{a}$ denote the chemical potential, number of particles, and Hamiltonian of the system, respectively; the summation is carried out over all particle configurations (here and henceforth we use the system of units with $k_{B} T$ =1). The Hamiltonian and number of particles are given by

$$
H_{a}=-\varepsilon_{d} \sum_{i \in d} n_{i}-\varepsilon_{s} \sum_{i \in S} n_{i}+\varphi \sum_{\langle n n\rangle} n_{i} n_{j}, \quad N_{a}=\sum_{i=1}^{N} n_{i} .
$$

Here $\varphi$ is the pair interaction energy of the nearest-neighbor $(\mathrm{NN})$ particles, and symbols $i \in d, i \in s,\langle\mathrm{NN}\rangle$ denote summation over all $d$ sites, $s$ sites, and lattice bonds, respectively.

The migration of particles over the lattice is described by some diffusion coefficients. The tracer diffusion coefficient $D_{t}$ addresses the random walk of individual tagged particles, i.e.,

$$
D_{t}=\lim _{t \rightarrow \infty} \frac{1}{2 d N_{a} t} \sum_{i=1}^{N_{a}}\left\langle\left[\vec{r}_{i}(t)-\vec{r}_{i}(0)\right]^{2}\right\rangle .
$$

Here $\vec{r}_{i}(t)$ denotes the displacement of the $i$ th particle as a function of time $t ; d$ is the dimensionality of the system; the angular brackets $\langle\cdots\rangle$ symbolize the ensemble average. Another useful quantity is the jump diffusion coefficient, $D_{j}$, related to the asymptotic behavior of the center of mass of the system, 


$$
D_{j}=\lim _{t \rightarrow \infty} \frac{1}{2 d N_{a} t}\left\langle\left\{\sum_{i=0}^{N_{a}}\left[\vec{r}_{i}(t)-\vec{r}_{i}(0)\right]\right\}^{2}\right\rangle .
$$

The definitions for $D_{t}$ and $D_{j}$ are well-suited for MC simulations as they are expressed in terms of directly accessible quantities.

The chemical diffusion coefficient $D_{c}$ is determined by Fick's first law, which constitutes the relationship between the flux of particles, $\vec{J}$, and the gradient of the particle density, $\theta$,

$$
\vec{J}=-D_{c} \vec{\nabla} \theta
$$

The chemical surface diffusion coefficient is of significance for the description of mass-transfer processes in the system.

To describe particle diffusion, the diffusion process must be resolved into its elementary components. Particle transport may be described in terms of jumps of particles in a regular or random energy landscape. Particles jump over the potential energy pits or lattice sites. A particle in the $i$ th site can jump to any of its $z$ unoccupied NN sites. The jumping particle must surmount a potential barrier $E_{i f}$ separating the initial and final sites. In the simplest case of the Langmuir lattice gas $(\varphi=0)$, the barrier is a constant $\varepsilon_{d}$ or $\varepsilon_{s}$ depending on the type of initial site. For interacting particles, the activation energy depends on the number of adjacent particles. We assume that the interactions affect the minima of the potential landscape and neglect the influence on an activated particle at the saddle point of the potential barrier. Then, the jump frequency from the $i$ th to the $f$ th site has the following form:

$$
\nu_{i f}=\nu \exp \left\{-\varepsilon_{i}+\varphi \sum_{k=1}^{z} n_{k}\right\},
$$

where summation runs over all NN's of the $i$ th site. The jump frequency depends on the energy of the initial state only and is the same for jumps to all NN sites. The model should be appropriate for a short-range lateral interaction between the particles.

Next we consider the evolution of the occupation numbers. The balance equation can be written in the following form:

$$
n_{i}(t+\Delta t)-n_{i}(t)=\sum_{f=1}^{z}\left[J_{f i}(\Delta t)-J_{i f}(\Delta t)\right]
$$

where $J_{i f}(\Delta t)$ denotes the number of particle jumps from the $i$ th to the $f$ th site during the time interval $\Delta t$. Invoking the local equilibrium approximation and neglecting memory effects, an analytical expression for $D_{c}$ can be derived (for details, see Refs. 1, 3, 7, and 8). Utilizing these approximations, the balance equation is easily reduced to the ordinary diffusion equation with the chemical diffusion coefficient given by

$$
D_{c}=D_{0} \exp (\mu) P_{00} / \chi_{T},
$$

where $D_{0}$ denotes the diffusion coefficient for one particle on the empty lattice; the correlation function $P_{00}$ describes the probability to find a pair of empty NN sites,

$$
P_{00}=\left\langle h_{i} h_{f}\right\rangle \equiv\left\langle\left(1-n_{i}\right)\left(1-n_{f}\right)\right\rangle,
$$

and $\chi_{T}$ is the isothermal susceptibility or the mean-square particle density fluctuations,

$$
\chi_{T}=\frac{1}{N} \sum_{i, j}\left(n_{i}-\theta\right)\left(n_{j}-\theta\right) .
$$

The jump and chemical diffusion coefficients are simply related via the Kubo-Green equation

$$
D_{c}=\theta D_{j} / \chi_{T} .
$$

The thermodynamic quantities that occur on the right-hand side of Eq. (9) can be expressed via the first and second derivatives of the free energy over its arguments as follows:

$$
\theta=\frac{\partial F}{\partial \mu}, \quad \chi_{T}=\frac{\partial^{2} F}{\partial \mu^{2}}, \quad P_{00}=1-2 \theta-\frac{2}{z}\left(\frac{\partial F}{\partial \varphi}\right) .
$$

Note that the kinetic coefficients $\left(D_{c}\right.$ and $\left.D_{j}\right)$ that describe, in principle, the nonequilibrium behavior of the lattice-gas system are related to the equilibrium thermodynamic quantities.

The density and temperature dependencies of the diffusion coefficient can be compared with the corresponding data obtained by the MC method. ${ }^{7,9-13}$ There is a very good coincidence of the data obtained by these conceptually different methods over wide temperature and density regions as well as different lateral interactions and lattice symmetries. But this expression works badly for inhomogeneous lattices with different kinds of adsorption sites. Qualitative discrepancies arise when the site inhomogeneity cannot be neglected, i.e., when $\exp \left(\varepsilon_{d}\right) \gg \exp \left(\varepsilon_{s}\right)$. In the following, we extend the derivation of analytical expressions for the chemical and jump diffusion coefficients.

Let us consider the particle migration on an inhomogeneous lattice in detail. All particles tend to occupy $d$ sites resulting in either an almost empty $s$ sublattice and partially filled $d$ sublattice $\left(\theta<\frac{1}{2}\right)$, or a completely filled $d$ sublattice and partially filled $s$ sublattice $\left(\theta>\frac{1}{2}\right)$. The inhomogeneity implies a higher jump probability for an $s \rightarrow d$ jump than for a $d \rightarrow s$ jump, and a rather specific correlation between the particle jumps arises in this case: Any $d \rightarrow s$ jump transfers a particle to the $s$ sublattice and creates an unstable local nonequilibrium configuration consisting of a pair of neighboring sites with the $s$ site occupied and the $d$ site unoccupied. There are two fast decay channels for this configuration: either the particle jumps to any of the NN empty deep sites or the deep site is filled by a particle from any filled NN shallow sites. As (slow) creation and (fast) decay of the intermediate nonequilibrium configuration take place on largely different time scales, their combination ought to be considered as the central entity of the diffusion process. The particle jumps collect into pairs: Any $d \rightarrow s$ jump is followed immediately by an $s \rightarrow d$ jump. Such jump pairs are the most frequent events and they govern the particle migration. The 
frequency of this event is determined by its slowest component, the $d \rightarrow s$ jump frequency. Despite the fact that the individual jumps are statistically uncorrelated, the lattice inhomogeneity imposes a strong pairwise correlation between $d \rightarrow s$ and $s \rightarrow d$ jumps. Correlation between jump pairs is absent. This specific correlation appears at low temperatures and will be especially important at $\theta=\frac{1}{2}$ when all deep sites are occupied and all shallow sites are empty.

The characteristic density $\theta=\frac{1}{2}$ separates two regions with distinctly different types of jump pairs dominating the particle migration. For $\theta<\frac{1}{2}$, the $s$ sublattice is empty and any act of migration starts by a particle $d \rightarrow s$ jump from a deep (initial) to any empty NN shallow (intermediate) site. Shortly after an $s \rightarrow d$ jump, the particle leaves the intermediate site and moves to some empty (final) $d$ site. The $s \rightarrow d$ jump probability depends on the total number of empty NN $d$ sites, $n_{\text {occ }}$, i.e., $\frac{1}{z-n_{\text {occ }}}$, where $z$ denotes the coordination number of the lattice. The migration of the particles proceeds over the $d$ sublattice using the empty $s$ sites as the intermediate steps. The probability of the jump pair depends not only on the occupation numbers of the initial and final sites, but on the occupation numbers of the intermediate $\mathrm{NN}$ sites.

For $\theta>\frac{1}{2}$ the $d$ sublattice is filled and the $s$ sublattice is partially occupied. Again, any act of migration starts by a $d \rightarrow s$ jump from an (intermediate) $d$ site to a final empty shallow NN site. The jump creates a "hole" in the completely occupied $d$ sublattice and initiates fast $s \rightarrow d$ jumps from some (initial) shallow NN site to the intermediate $d$ site. The $s \rightarrow d$ jump probability depends upon the number of occupied NN sites, $n_{\text {occ }}$, at the intermediate $d$ site, i.e., $\frac{1}{n_{\text {occ }}}$, where $n_{\text {occ }}<z$. The pair of successive $d \rightarrow s$ and $s \rightarrow d$ jumps causes particles to migrate over the $s$ sublattice using $d$ sites as intermediate steps.

The $s \rightarrow d$ jump probability determines the total probability of backward jumps, where initial and final sites are identical. The total probability of the backward jump is increased relative to all other possibilities as either the initial $\left(\theta<\frac{1}{2}\right)$ or final site $\left(\theta<\frac{1}{2}\right)$ is always unoccupied and occupied, respectively. There is some similarity to the backward correlation of the motion of tagged particles. ${ }^{14}$

Summing up contributions from all possible particle configurations, we obtain the balance equation for the evolution of the occupation numbers. Using the same approximations as in the case of a homogeneous lattice, the expression for the chemical diffusion coefficient runs as follows:

$$
D_{c}(\theta)=D_{c}(0) \exp (\mu) P(z) \chi_{T}^{-1},
$$

where the correlation function $P(z)$ is given by

$$
\begin{aligned}
P(z)= & \frac{1}{z-1}\left\langleh _ { 1 } h _ { c } \left( z-1-n_{2}\right.\right. \\
& \left.\left.-n_{2} n_{3}-\cdots-n_{2} n_{3} \cdots n_{z}\right)\right\rangle, \quad \theta<1 / 2, \\
P(z)= & \frac{1}{z-1}\left\langleh _ { 1 } h _ { c } \left( z-1-h_{2}\right.\right. \\
& \left.\left.-h_{2} h_{3}-\cdots-h_{2} h_{3} \cdots h_{z}\right)\right\rangle, \quad \theta>1 / 2 .
\end{aligned}
$$

Here $h_{i} \equiv 1-n_{i} ; c$ denotes the intermediate site and the sub- scripts $1, \ldots z$, its NN's. For details of the derivation, see the Appendix. It should be noted that the upper and lower expressions for $P(z)$ are exponentially small outside the regions of their definition. Then, one can use the sum of these expressions for the calculations of the diffusion coefficients.

Equation (14) is rather universal and applies to many lattices of different symmetries and dimensionality such as a one-dimensional chain, two-dimensional (square and honeycomb), and three-dimensional cubic inhomogeneous lattices. The expression is more complex as compared with the similar expression Eq. (9). It depends on the correlation functions of clusters of $2,3, \ldots, z+1$ sites. These correlators can be approximately expressed via the first derivative of the free energy over the interaction parameter and mean values of the occupation numbers on the deep and shallow sublattices.

\section{REAL-SPACE RENORMALIZATION-GROUP TRANSFORMATION}

A variety of analytical methods can be used for the calculations of the thermodynamical quantities that enter the expressions for the diffusion coefficients. Among them, the RSRG approach is particularly simple so that a rather modest effort suffices to compute data of good accuracy. This applies especially to the two-dimensional lattice-gas systems.

In this section, we will briefly outline the RSRG method used for this purpose. It is well known that there is a one-toone correspondence between the lattice-gas model and the Ising spin model in an external magnetic field. Empty and occupied sites are equivalent to $s=-1$ and $s=1$, respectively. Using the obvious relation between the site spins and occupation numbers, $2 n_{i}=1+s_{i}$, the equivalent reduced Hamiltonian of the Ising model runs

$$
H(s)=h_{d} \sum_{i \in d} s_{i}+h_{s} \sum_{i \in s} s_{i}+k \sum_{\langle n n\rangle} s_{i} s_{j}+N c .
$$

Here

$$
\begin{gathered}
h_{d}=\left(\mu+\varepsilon_{d}-\varphi z / 2\right) / 2, \\
h_{s}=\left(\mu+\varepsilon_{s}-\varphi z / 2\right) / 2, \\
k=-\varphi / 4, \\
c=\left(2 \mu+\varepsilon_{d}+\varepsilon_{s}-\varphi z / 2\right) / 4 .
\end{gathered}
$$

The thermodynamic properties of the lattice gas are described by the magnetic behavior of the spin system. Although Eqs. (3) and (16) are fully equivalent, we prefer to use the spin representation in this section because of its apparent symmetry with respect to the magnetic field. However, we will refer to lattice-gas terms where this seems to be more transparent. The spin model looks slightly strange as there are two magnetic fields $h_{d}$ and $h_{s}$ acting separately on the spins in the $d$ and $s$ sublattices. The fields have different values and even directions depending on the relation between the chemical potential and site depths difference $\varepsilon_{d}$ $-\varepsilon_{s}$.

The case $\varphi<0$ corresponds to ferromagnetic (F) spin-spin interaction and $\varphi>0$ describes the antiferromagnetic (AF) 
spin model. Whereas $\mathrm{F}$ interaction aligns all spins up or down, AF interaction aligns spins on different sublattices in the opposite directions.

In the RSRG method developed by Niemeyer and van Leeuwen ${ }^{15}$ and Nauenberg and Nienhuis, ${ }^{16,17}$ the whole lattice is divided into blocks (or cells) of $L$ sites. ${ }^{18,19}$ A block spin $S_{\alpha}$ is assigned to each block. All blocks together must form a lattice of the same symmetry. The RSRG transformation of the spin system allows the reduction of the number of independent variables, i.e., the transition from the set of $N$ site spins $\left\{s_{i}\right\}$ to $N / L$ block spins $\left\{S_{\alpha}\right\}$. For blocks with an odd number of spins $L$, the block spin $S_{\alpha}$ is usually determined by the so-called "majority rule," 18

$$
S_{\alpha}=\operatorname{sgn}\left(\sum_{i=1}^{L} s_{i}\right), \quad \text { where } \operatorname{sgn}(x)= \begin{cases}+1 & \text { if } x>0 \\ -1 & \text { if } x<0 .\end{cases}
$$

The main goal of any RSRG transformation of the Hamiltonian Eq. (16) is to obtain the result in the same form as the original Hamiltonian plus possibly some insignificant terms, weakly affecting the critical behavior of the system. In the framework of the RSRG approach, one usually employs periodic boundary conditions. It is assumed that the whole lattice is given by the periodic continuation of a small cluster of blocks. In the present work, we consider the smallest possible cluster of two blocks (see Fig. 1). Due to the simplicity of this cluster, no additional interactions appear in the renormalized Hamiltonian. It is the same Hamiltonian of the square lattice of spins with, however, renormalized values for the external magnetic fields $h_{d}^{(1)}, h_{s}^{(1)}$ and spin-spin interaction parameter $k^{(1)}$,

$$
\begin{aligned}
h_{d}^{(1)} & S_{1}+h_{s}^{(1)} S_{2}+4 k^{(1)} S_{1} S_{2}+2 L g \\
& =\ln \left\{\sum_{\{s\}} P(S, s) \exp [H(s)]\right\}=\Psi\left(S_{1}, S_{2}\right) .
\end{aligned}
$$

Here the summation is carried out over all possible configurations $\left\{s_{i}\right\}$ for fixed values of the block spins $S_{1,2}$. The relations between the renormalized and original values of the parameters are given by the following system of RG equations:

$$
\begin{aligned}
h_{d}^{(1)}\left(h_{d}, h_{s}, k\right)= & \frac{1}{4}[\Psi(1,1)-\Psi(-1,-1)+\Psi(1,-1) \\
& -\Psi(-1,1)], \\
h_{s}^{(1)}\left(h_{d}, h_{s}, k\right)= & \frac{1}{4}[\Psi(1,1)-\Psi(-1,-1)-\Psi(1,-1) \\
& +\Psi(-1,1)], \\
k^{(1)}\left(h_{d}, h_{s}, k\right)= & \frac{1}{16}[\Psi(1,1)+\Psi(-1,-1)-\Psi(1,-1) \\
& -\Psi(-1,1)],
\end{aligned}
$$

$$
\begin{aligned}
g\left(h_{d}, h_{s}, k\right)= & \frac{1}{8 L}[\Psi(1,1)+\Psi(-1,-1)+\Psi(1,-1) \\
& +\Psi(-1,1)] .
\end{aligned}
$$

The RSRG transformation functions $\Psi( \pm 1, \pm 1)$ depend strongly on the size and the symmetry of the blocks. They have the following general form:

$\Psi( \pm 1, \pm 1)=\ln \left\{\sum_{i=1}^{N_{ \pm \pm \pm}} a_{i}^{( \pm, \pm)} \exp \left[h_{d} b_{i}^{( \pm, \pm)}+h_{s} c_{i}^{( \pm, \pm)}+k d_{i}^{( \pm, \pm)}\right]\right\}$.

For the cluster of two "crosses" with $L=17$, shown in Fig. 1, the numbers of terms in the transformation functions are the following: $N_{1,1}=N_{-1,-1}=2669$ and $N_{1,-1}=N_{-1,1}=3781$. The four four-column tables (matrices) $\left\{a_{i}^{ \pm \pm}, b_{i}^{ \pm \pm}, c_{i}^{ \pm \pm}, d_{i}^{ \pm \pm}\right\}$are very useful for fast calculations of the free energy and its derivatives for any values of the magnetic field and particle interaction (temperature).

As was shown by Nauenberg and Nienhuis, ${ }^{16}$ the free energy of the system for any values of magnetic fields and interaction parameter $F\left(h_{d}, h_{s}, k\right)$ can be evaluated in the series of sequential RSRG transformations of the original Hamiltonian,

$$
F\left(h_{d}, h_{s}, k\right)=c+\sum_{m=0}^{n} L^{-m} g\left(h_{d}^{(m)}, h_{s}^{(m)}, k^{(m)}\right) .
$$

Here $h_{d}^{(m)}, h_{s}^{(m)}, k^{(m)}$ are the parameters of the $m$ th RSRG transformation; $h_{d, s}^{(0)}=h_{d, s}, k^{(0)}=k$.

\section{MONTE CARLO SIMULATIONS OF THE SURFACE DIFFUSION}

The Monte Carlo technique is one of the most universal and reliable methods that is used widely to study complex phenomena, especially when analytical approaches are not available or work badly. Due to its simplicity and the availability of powerful supercomputers, MC simulations have been widely used for investigations of kinetic properties of lattice-gas models. We applied the MC approach to calculations of the tracer and jump diffusion coefficient and some thermodynamic quantities of the particle system.

For a detailed description of MC application in the field of the surface diffusion, the interested reader is referred to Refs. 20-23. Here we give only a brief overview of the MC algorithms used for simulations.

The system represented by the Hamiltonian Eq. (3) is realized by a two-dimensional array of $N=M^{2}$ sites $(M=32,64)$ with periodic boundary conditions applied. On a lattice of $M^{2}$ sites, the nearest neighbors of a site with coordinates $(i, j)$ are $((i \pm 1) \bmod M, j)$ and $(i,(j \pm 1) \bmod M)$, where $\bmod M$ denotes remainder modulo $M$. This ensures the equivalence of all sites on the lattice. Choosing $M$ as powers of 2, the modulo operation can be replaced by the corresponding bit operations $i \bmod M=\operatorname{IAND}(i, M-1)$. An alternative is the so-called helical boundary conditions (HBC) ${ }^{24}$ An example of HBC for a lattice with $M=4$ is 


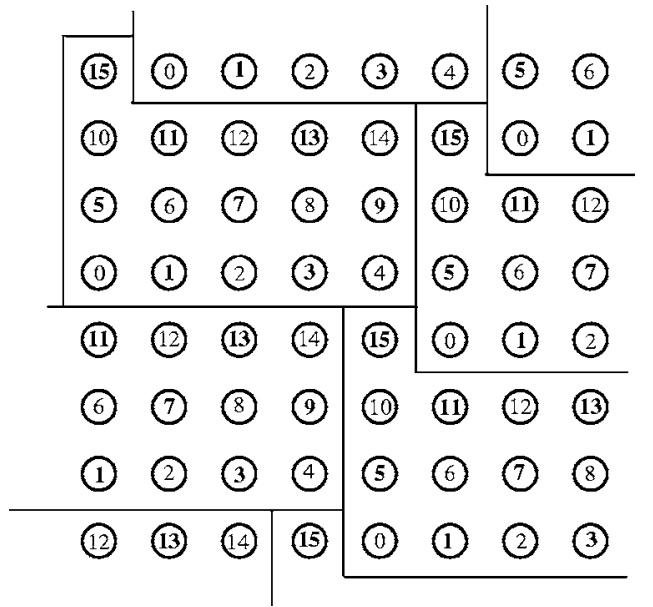

FIG. 2. An example of the HBC. Square lattice is obtained by the periodic continuation of a small cluster of 16 sites. The bigger clusters are constructed in the same way.

plotted in Fig. 2. Due to the heterogeneous surface relief, we cannot use simple square original $M^{2}$ arrays displaced by one row in the vertical direction to build the infinite lattice. Instead of this, we use $(M+1) \times(M-1)$ rectangles with an additional lattice site glued at the left upper corner. Its looks may be odd, but nevertheless this representation offers computational advantages: (i) all lattice sites are represented by a one-dimensional index $i$ varying from 0 to $M^{2}-1$. (ii) The NN's of the $i$ th site are determined by fast bitwise operations $\operatorname{IAND}\left(i \pm 1, M^{2}-1\right)$ and $\operatorname{IAND}\left(i \pm(M+1), M^{2}-1\right)$, and the kind of the site (deep or shallow) is determined by the parity of its index.

An initial configuration is generated by adsorbing $N_{a}$ $=\theta N$ particles on the surface at random. The saddle point energy (describing the wells that need to be overcome by diffusing particles) is equal to zero. It is assumed that the saddle point energy is not affected by the particle-particle interactions. The jump probability $P_{i}$ is given by

$$
P_{i}=\kappa \exp \left(-E_{i}\right),
$$

with $\kappa$ as the normalization factor and $E_{i}$ denotes the activation energy for such a jump, i.e., the energy difference between the saddle point and the energy of the initial site, the latter being influenced by the NN interaction,

$$
E_{i}=\varepsilon_{i}-\varphi \sum_{k} n_{k} .
$$

$\kappa$ is chosen as $\kappa=\exp \left(\min \left\{E_{i}\right\}\right)$.

The ordinary MC algorithm may be described as follows. First, an initial site $i$ of the whole lattice is picked at random. If filled, an adjacent final site $f$ is randomly selected. If the destination is vacant, a third random number $\eta$ is generated. A jump occurs if $\eta \leqslant P_{i}$. One MC step (MCS) corresponds to $N$ repetitions of the jump algorithm. The main drawback of this $\mathrm{MC}$ algorithm for inhomogeneous lattices is a very small transition probability for jumps from $d$ sites. A large part of the computing time is spent in attempting jumps that are rejected. To overcome this difficulty, we use the BKL algorithm, after Bortz, Kalos, and Lebowitz. ${ }^{25}$ It operates in the space of possible events instead of the real space as the ordinary MC algorithm does. There are no rejected attempts, which results in a high efficiency of the BKL algorithm. It has been described in detail elsewhere. ${ }^{26,27}$ The BKL algorithm picks up an event from the common space of possible events at random. All possible events are grouped into classes of events of the same probability. In order to avoid the time-consuming maintenance of the event list, we note that at a given particle distribution all events from a given site belong to the same event class, so that the number of possible events is computable from the class index. Hence, it is sufficient to maintain the association between sites and classes. Technically, four random numbers are required in order to select the time interval between successive jumps, the event class, the site belonging to this class, and the direction of jump (if necessary). For efficient MC simulations, we note that the number of MC jumps in one MCS cycle required to suppress correlations between MCS configurations depends upon the particle density. Correspondingly, for the BKL algorithm, the accumulated time difference can be used as a termination criterion of an MCS cycle. Choosing this criterion density-dependent offers a possibility for very substantial savings in computer time without affecting the results.

We used the MC simulations for calculations of the tracer and jump diffusion coefficients, adsorption isotherms, and isothermal susceptibility. The chemical diffusion coefficient has been determined via the Kubo-Green relation, here written as

$$
D_{c}=\theta D_{j} \frac{N_{p}\left(N-N_{p}\right)}{N\left\langle(\delta n)^{2}\right\rangle},
$$

where $N_{p}$ is the number of sites inside the probe area, and $\left\langle(\delta n)^{2}\right\rangle$ is the mean-square fluctuation of the number of particles in this area. ${ }^{28}$ The shape of the probe region may be arbitrary, square, or rectangular.

The dependencies $\mu(\theta)$ have been calculated using the method of local states. ${ }^{29}$ The idea of the method is rather simple. The energy of any particle can take the $2(z+1)$ values: $E_{i}=-\varepsilon_{d, s}+n \varphi, n=0,1,2, \ldots, z$, depending on the number of NN's. The energies define the local states of the particles. The set of conjugate states is determined for holes (empty sites). All conjugate states have the same zero energy. The frequencies of occurrence (ensemble average populations) of the $i$ th local state and its conjugation are denoted as $\sigma_{i}$ and $\sigma_{i}^{\prime}$. They are related by the condition of the detailed balance as follows:

$$
\mu=\ln \left(\sigma_{i} / \sigma_{i}^{\prime}\right)+E_{i}, \quad i=1, \ldots, 2(z+1) .
$$

To smooth out the fluctuations, it is advisable to average $\mu$ over the most probable local states and exclude rare configurations.

The MC codes have been parallelized using MPI by dividing the entire workload of coverage-dependent configuration sampling into independent tasks, so that the overall communication load is negligible. We have averaged over 40 000-160 000 configurations, depending upon the lateral 


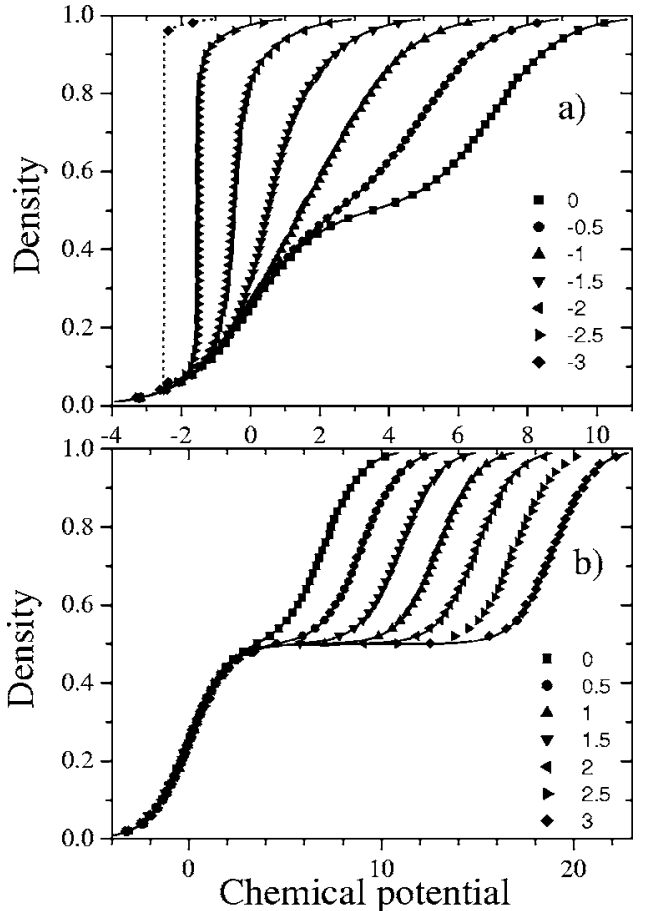

FIG. 3. Adsorption isotherms $\theta$ vs $\mu$ for different values of the interaction parameter $\varphi$ as indicated. Lines and symbols denote the RSRG and MC data.

interaction strength $\varphi$. The accuracy has been judged by monitoring the change of computed quantities as a function of the number of configurations and the MCS cycle termination criterion. The final results are almost indistinguishable on the scale of the figures.

\section{RESULTS AND DISCUSSION}

We have calculated using the RSRG and MC methods the adsorption isotherms, pair correlation function, isothermal susceptibility, tracer, jump, and chemical diffusion coefficients for attractive and repulsive lateral interaction between the particles adsorbed on an inhomogeneous square lattice. The inhomogeneity parameter $\varepsilon_{d}-\varepsilon_{s}$ is the same for all calculations and equal to 7 .

In Fig. 3, we plot the adsorption isotherms for some representative values of the lateral interaction parameter $\varphi$. The agreement between the RSRG results and MC data is excellent over the entire temperature and coverage range for attractive and repulsive interactions. At high temperature $(\varphi \rightarrow 0)$, the isotherms are close to the Langmuir case,

$$
\theta_{L}(\mu)=\frac{1}{2}\left[\frac{\exp \left(\mu+\varepsilon_{d}\right)}{1+\exp \left(\mu+\varepsilon_{d}\right)}+\frac{\exp \left(\mu+\varepsilon_{s}\right)}{1+\exp \left(\mu+\varepsilon_{s}\right)}\right]
$$

If the temperature decreases, the effects of the heterogeneity and lateral interaction appear on the dependencies. It should be noted that the inhomogeneity plays a rather specific role: it cancels lateral repulsion between the particles at low coverage $\left(\theta<\frac{1}{2}\right)$, placing them on the $d$-sublattice sites. Then, the particles do not "feel" each other. The dependencies for all quantities are very close to those obtained for the Lang-

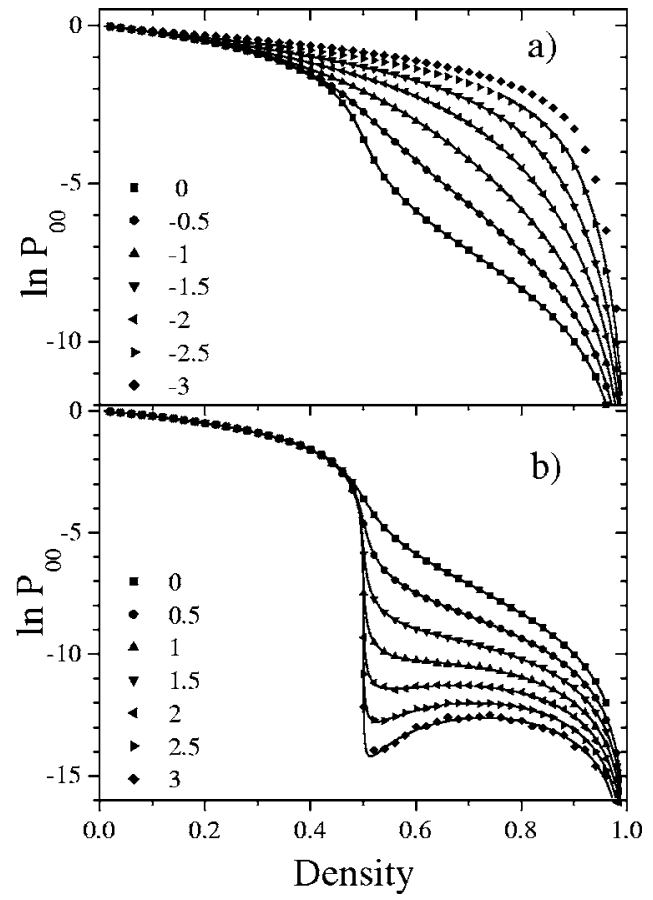

FIG. 4. The pair correlation function $\ln P_{00}$ vs $\theta$. Notations are the same as in Fig. 3.

muir case in this region, despite the different values of the lateral interaction parameter $\varphi$.

Strong repulsion results in a broad, almost horizontal plateau at $\theta=\frac{1}{2}$ [see Fig. 3(b)]. It corresponds to a stable, ordered structure when particles occupy the $d$ sublattice only. Attraction produces another type of singular behavior. As temperature is decreased, the dependencies become steeper and at critical temperature the tangent becomes vertical. This means there is an infinite growth of the isothermal susceptibility, which is the second derivative of the free energy over $\mu$. If the temperature decreases below critical, the isotherms exhibit a discontinuity, i.e., jumps of the particle density [shown in (a) by the vertical dashed line]. This discontinuity corresponds to the first-order phase transition. There are two critical values of the particle density, $\theta_{c}$ and $1-\theta_{c}$, corresponding to a single value of the chemical potential $\mu_{c}$. The region $\left(\theta_{c}, 1-\theta_{c}\right)$ is inaccessible for the RSRG method. Therefore, we have only a few RSRG data for some dependencies [some lines in (a) consist of two small parts for $\theta<\theta_{c}$ and $\left.\theta>1-\theta_{c}\right]$.

The pair correlation function $P_{00}$ describes the probability to find two holes on nearest-neighbor sites. At high temperatures, $P_{00}$ is nicely represented by its mean-field dependence $P_{00}^{\mathrm{MF}} \equiv\left(1-\theta_{d}\right)\left(1-\theta_{s}\right)$. At low temperatures, the dependence is well described by the line: $P_{00} \approx 1-2 \theta$ for $\theta<\frac{1}{2}$ and $P_{00} \rightarrow 0$ for $\theta>\frac{1}{2}$.

To show clearly the coincidence between the RSRG and MC data, we plotted the dependencies $\ln P_{00}(\theta)$ in Fig. 4. Again, the agreement between lines (RSRG) and symbols (MC) is ideal. The isotherms and pair correlation functions are the first derivatives of the free energy over its arguments. These quantities do not have singularities and their behavior is described by the RSRG method with high accuracy. 


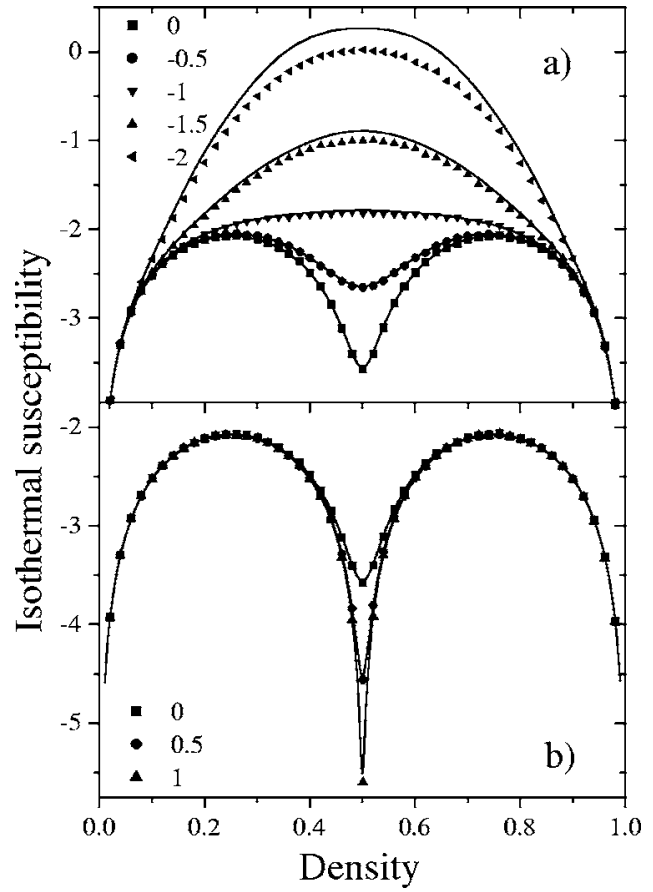

FIG. 5. Isothermal susceptibility $\chi_{T}$ vs $\theta$. Lines denote RSRG results, symbols denote the MC data.

The calculations of the diffusion coefficients requires knowledge of the correlation functions, which cannot be obtained by the RSRG method. These are the probabilities $\left\langle h_{c} h_{1} h_{2}\right\rangle,\left\langle h_{c} h_{1} h_{2} h_{3}\right\rangle, \ldots,\left\langle h_{c} h_{1} \cdots h_{z}\right\rangle$, to find clusters of $3, \ldots, z+1$ empty sites with one (central) site, which belongs to the $d$ or $s$ sublattice, and others are its NN's. For the Langmuir lattice gas, the probabilities are simply powers of corresponding hole densities,

$$
\begin{gathered}
\left\langle h_{c} h_{1} \cdots h_{k}\right\rangle \equiv\left(1-\theta_{d}\right)\left(1-\theta_{s}\right)^{k}, \quad c \in d, \\
\left\langle h_{c} h_{1} \cdots h_{k}\right\rangle \equiv\left(1-\theta_{s}\right)\left(1-\theta_{d}\right)^{k}, \quad c \in s .
\end{gathered}
$$

Such approximation is rather good for $\theta<\frac{1}{2}$, where the particles can be considered as noninteractive. But for higher densities it gives noticeable discrepancies. In this case, we expressed all necessary functions via the simplest two-site correlator we have at hand, $P_{00}$, as follows:

$$
\begin{aligned}
& \left\langle h_{c} h_{1} \cdots h_{k}\right\rangle \approx P_{00}^{k}\left(1-\theta_{d}\right)^{1-k}, \quad c \in d, \\
& \left\langle h_{c} h_{1} \ldots h_{k}\right\rangle \approx P_{00}^{k}\left(1-\theta_{s}\right)^{1-k}, \quad c \in s .
\end{aligned}
$$

This approximation works very well over the entire temperature and density range.

The quantity most sensitive to the method of calculation is the isothermal susceptibility - the mean square density fluctuations. The coverage dependencies of the isothermal susceptibility are plotted in Fig. 5. At high temperature, the fluctuations are well described by its mean-field expression,

$$
\chi_{T}^{\mathrm{MF}}=\left[\theta_{d}\left(1-\theta_{d}\right)+\theta_{s}\left(1-\theta_{s}\right)\right] / 2 .
$$

The lateral repulsion decreases the density fluctuations keeping particles apart from each other. The density fluctuations

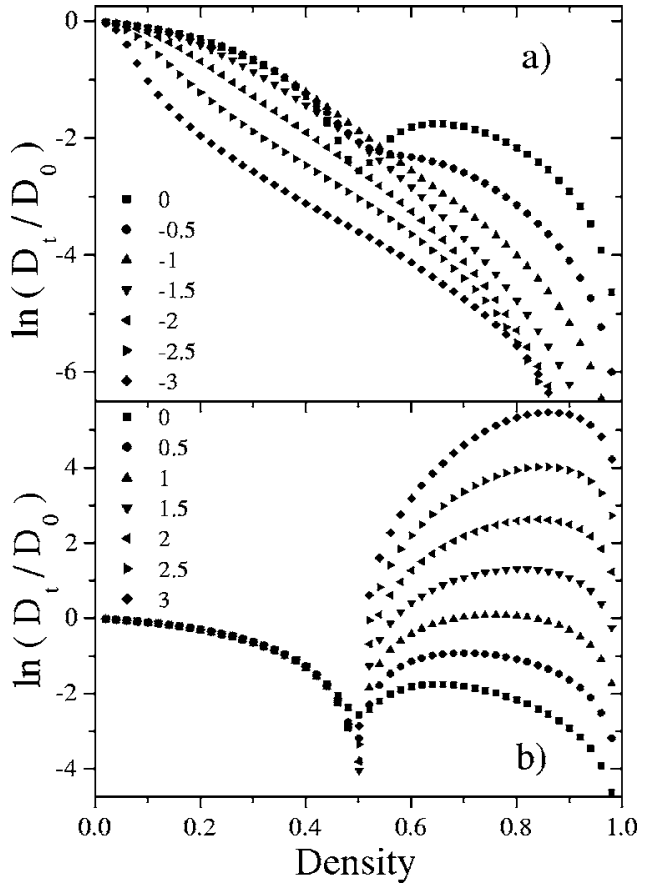

FIG. 6. The density dependencies of the normalized tracer diffusion coefficient for repulsive and attractive interactions. The MC data only.

are strongly suppressed at $\theta=\frac{1}{2}$ [see Fig. 5(b)]. In the ordered phase, any density disturbance (i.e., displacement of a particle from its "right" position in the filled $d$ sublattice to any site of the empty $s$ sublattice) increases significantly the energy of the system and is thermodynamically unfavorable. As the density does not equal $\frac{1}{2}$, there are fluctuations of the nonstoichiometric nature that do not require additional energy for their existence and cannot be removed by the particle jumps. Therefore, $\chi_{T}$ increases when $\theta$ deviates from the stoichiometric value and the dependencies $\chi_{T}(\theta)$ have a deep and narrow minimum at low temperatures but remains finite and analytical in this point. The coincidence between the RSRG and MC data in Fig. 5(b) is rather good even at low temperatures.

Strong attraction decreases relaxation of the density disturbances. Any coverage fluctuation decays slower and slower as the lateral interaction approaches its critical value. The system becomes unstable at the critical point and fluctuations diverge to infinity.

The coincidence between the RSRG and MC data in Fig. 5(a) is very good at high temperatures, but there are visible and regular deviations between the RSRG and MC data that grow as temperature is lowered. It should be noted that irrespective of the method, the isothermal susceptibility is the most challenging property to compute. Due to its singular behavior, the data are more sensitive to the choice of the RSRG blocks. The (infinite) growth of the density fluctuations is controlled by the critical exponents, so the proximity to the exact values becomes important.

The coverage dependencies of the tracer diffusion coefficient are shown in Figs. 6(a) and 6(b) for repulsive and attractive interaction. It has already been mentioned that the 


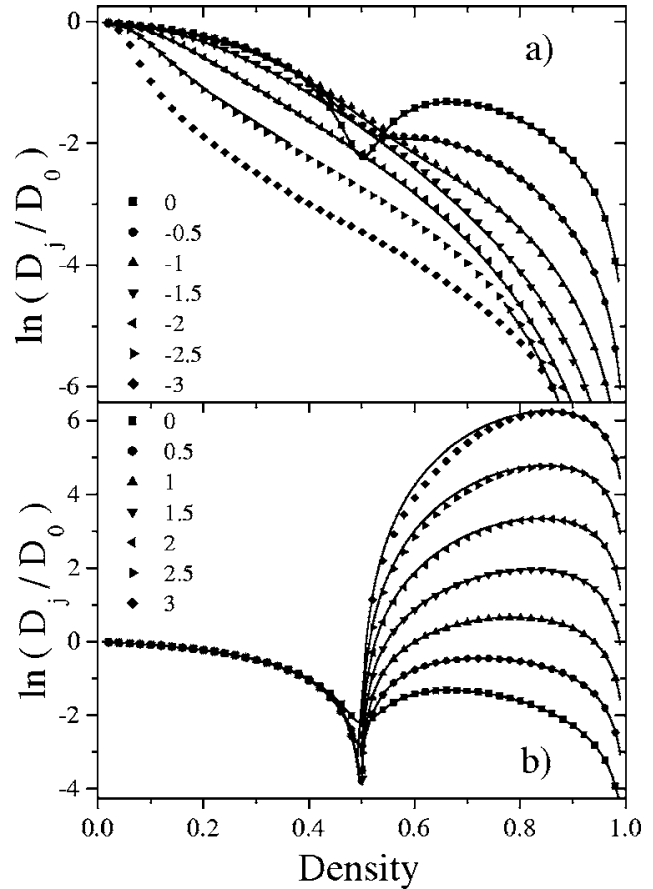

FIG. 7. The density dependencies of the normalized jump diffusion coefficient. Solid lines are calculated using the model of jump pairs.

repulsion accelerates particle migration on the surface, as is clearly seen in Fig. 6. The dependencies for the tracer diffusion coefficient $D_{t}$ were obtained by the MC simulations only. We have no analytical expression for the tracer diffusion coefficient. The tracer diffusion coefficient describes diffusion of a single tagged particle. At high temperatures, $D_{t}$ is a monotonic decreasing nonlinear function of the surface coverage. As temperature is lowered, the tracer diffusion coefficient shows a pronounced minimum at half monolayer coverage. The nature of this minimum can be explained as follows. At low temperature, particles form perfect ordered structure at the stoichiometric density $\theta=\frac{1}{2}$. The overwhelming majority of jumps will be unsuccessful. Particles jump to the shallow sites and return back because all deep sites are occupied and all shallow sites are empty. Any deviation from the stoichiometric value will increase the particle diffusivity. Decreasing of the density causes holes in the $d$ sublattice, and particles situated close to a hole can occupy this hole by performing a pair of jumps. The increase of the density creates some particles in the $s$ sublattice. Then the particles in the $d$ sites situated close to this excess particles have some probability to perform a successful jump. Therefore, the tracer and jump diffusion coefficients grow when the density deviates from $\theta=\frac{1}{2}$.

The dependencies for the jump diffusion coefficient $D_{j}$ are plotted in Fig. 7. At high temperatures, the jump diffusion coefficient is a linear function of coverage $D_{j}=D_{0}(1$ $-\theta)$. As the temperature is lowered, a deep minimum is found at $\theta=\frac{1}{2}$ similar to that for the tracer diffusion coefficient. Strong repulsion results in a broad and high maximum for $\theta>\frac{1}{2}$. Again, RSRG and MC data coincide rather well. Only small deviations appear on the coverage dependencies for strong lateral interaction $(|\varphi|=2.5,3)$.

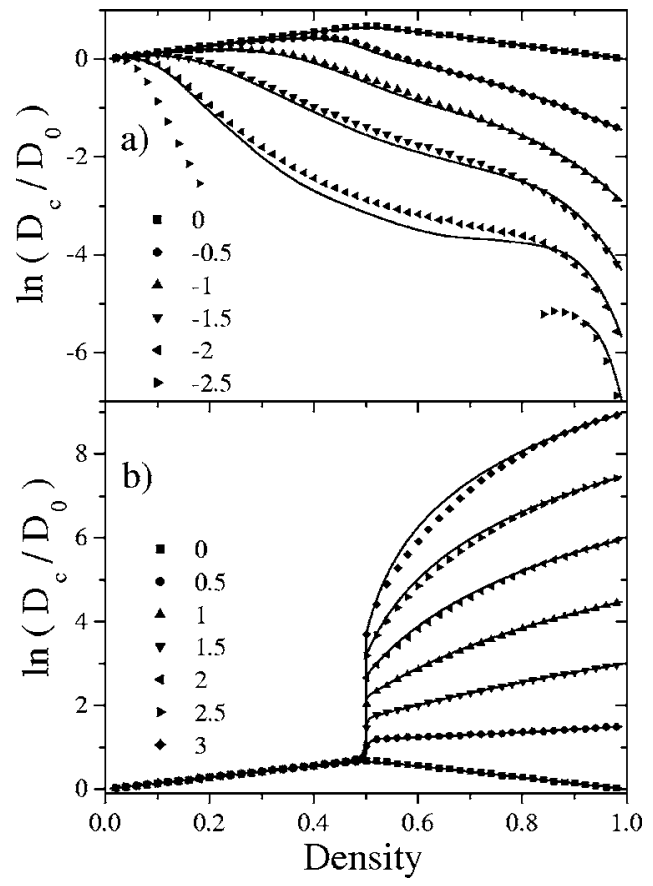

FIG. 8. The density dependencies of the normalized chemical diffusion coefficient. Solid lines are calculated using the model of jump pairs.

The dependencies for the chemical diffusion coefficient $D_{c}$ are shown in Fig. 8. There is a rather strong effect of the lateral interaction on the particle diffusion. The chemical diffusion coefficient grows from its initial value $D_{0}$ almost linearly for $\theta<\frac{1}{2}$ and then jumps at $\theta=\frac{1}{2}$. At this density, the lateral interaction is switched on and particles begin to "feel" each other. The chemical diffusion coefficient grows very fast if the density exceeds slightly the stoichiometric value.

In the limits of $\theta \rightarrow 0,1$, a jumping particle has either none or three nearest neighbors, respectively. Therefore, the limiting values of the diffusion coefficient are equal to

$$
\begin{gathered}
\lim _{\theta \rightarrow 0} D_{c}=D_{0}, \\
\lim _{\theta \rightarrow 1} D_{c}=D_{0} \exp [(z-1) \varphi] .
\end{gathered}
$$

Attraction inhibits the particle migration. The relaxation of the density disturbances became slower and slower as temperature decreases. The critical slowdown of the chemical diffusion coefficient is controlled by the singular behavior of the isothermal susceptibility. As was mentioned above, strong attraction causes the first-order phase transition. In this density region, the particle distribution is nonuniform. There are islands of dense phase with $\theta_{c} \rightarrow 1$ on the rarefied background with $\theta=1-\theta_{c}$. The islands grow and decay chaotically. The system is thermodynamically unstable. We cannot obtain any data by the RSRG method in this region.

The numerical data for the chemical diffusion coefficient are obtained using the Kubo-Green relation as a ratio of two quantities: jump diffusion coefficient and isothermal susceptibility. The good coincidence between the data obtained by 
the analytical and numerical methods means the applicability of the Kubo-Green relation for this model. Also it means that the approximate expressions for the correlation functions of higher orders via the pair correlation function $P_{00}$ are valid in wide density and temperature regions.

\section{SUMMARY}

We have investigated the diffusion of particles on inhomogeneous lattices with two kinds of sites. Such systems display specific peculiarities, which qualitatively affect the particle diffusion as compared to homogeneous lattices. Notably, the lattice inhomogeneity causes temperaturedependent specific correlation between particle jumps. This results in a qualitatively different nonmonotonic behavior of the diffusion coefficients: the appearance of a minimum $D_{t}(\theta)$ and $D_{j}(\theta)$.

The numerical BKL technique as well as the analytical RSRG method have been used to compute adsorption isotherms and the density dependencies of the pair correlation function, the isothermal susceptibility, the jump, tracer, and chemical diffusion coefficients at different temperatures. The excellent agreement between the data indicates that the RSRG method can be applied successfully for investigations of the thermodynamic properties of the lattice gas systems with strong lateral interactions.

The standard model of single jumps works rather well in simple lattice gas systems provided the lattice inhomogeneity is not substantial. However, the model is doomed to failure even at a qualitative level of accuracy in the case of inhomogeneous potential relief. An extension to the model of jump pairs has been proposed that forms a sound basis for the quantitatively correct description of the particle diffusion in such cases. This approach is quite general. The analytical expressions for the chemical diffusion coefficient derived on the basis of this model extension are valid for inhomogeneous lattices of different symmetry and dimensionality. The proposed model of jump pairs gives a simple and natural explanation of the peculiar characteristics of surface diffusion on inhomogeneous lattices mentioned above.

\section{ACKNOWLEDGMENTS}

This work has been supported by the Institutional Research Plan AV CR No. AV0Z 10100522, projects 1QS100100563 AV CR and 1M06002 MSMT CR. We gratefully acknowledge computer time on the IBM p690 supercomputer cluster at the John-von-Neumann Institute for Computing at the Research Centre Jülich where the Monte Carlo simulations have been carried out.

\section{APPENDIX}

Let us consider the first type of the jump pairs. A particle from the initial deep site (first) jumps to the final deep site (second) via the intermediate shallow $c$ th site. The total probability $J_{12}$ of the jump sequence is the sum of contributions coming from all possible particle configurations $n_{3}, n_{4}, \ldots, n_{z}$ with fixed $n_{c}=0, n_{1}=1$, and $n_{2}=0$,

$$
\begin{aligned}
J_{12} \propto & \frac{1}{z}\left\langle n_{1} h_{c} h_{2} h_{3} h_{4} \cdots h_{z}\right\rangle+\frac{1}{z-1}\left\langlen _ { 1 } h _ { c } h _ { 2 } \left( n_{3} h_{4} \cdots h_{z}\right.\right. \\
& \left.\left.+h_{3} n_{4} \cdots h_{z}+\cdots+h_{3} h_{4} \cdots n_{z}\right)\right\rangle \\
& +\frac{1}{z-2}\left\langlen _ { 1 } h _ { c } h _ { 2 } \left( n_{3} n_{4} \cdots h_{z}+n_{3} h_{4} n_{5} \cdots h_{z}+\cdots\right.\right. \\
& \left.+h_{3} h_{4} \cdots n_{z-1} n_{z}\right\rangle+\cdots+\frac{1}{2}\left\langle n_{1} h_{c} h_{2} n_{3} n_{4} \cdots n_{z}\right\rangle,
\end{aligned}
$$

which can be rewritten as

$$
\begin{aligned}
J_{12} \propto & \left\langlen _ { 1 } h _ { c } h _ { 2 } \left[ C_{z-2}^{0} z^{-1} h_{3} h_{4} \cdots h_{z}+C_{z-2}^{1}(z-1)^{-1} n_{3} h_{4} \cdots h_{z}\right.\right. \\
& \left.\left.+C_{z-2}^{2}(z-2)^{-1} n_{3} n_{4} \cdots h_{z}+\cdots+C_{z-2}^{z-2} 2^{-1} n_{3} n_{4} \cdots n_{z}\right]\right\rangle \\
\equiv & \sum_{p=1}^{z-1} \frac{(-1)^{p+1}}{(z-1)} C_{z-1}^{p}\langle n_{1} h_{\underbrace{}_{p}} \underbrace{n_{2} n_{3} \cdots}_{p}\rangle \sum_{n=0}^{p} \frac{(-1)^{n}}{z-n} C_{p}^{n}, \quad \text { (A2) }
\end{aligned}
$$

where binomial coefficients $C_{n}^{m}$ are defined as usual,

$$
C_{n}^{m}=\frac{n !}{(n-m) ! m !} .
$$

It is easy to see that the inner sum is equal to the definite integral as follows:

$$
\begin{aligned}
\sum_{n=0}^{p} \frac{(-1)^{n}}{z-n} C_{p}^{n} & =\sum_{n=0}^{p}(-1)^{n} C_{p}^{n} \int_{1}^{\infty} x^{n-z-1} d x \\
& =\int_{1}^{\infty} x^{-z-1}(1-x)^{p} d x .
\end{aligned}
$$

The substitution $x=1 / y$ reduces the integral to the standard form

$$
\begin{aligned}
\int_{1}^{\infty} x^{-z-1}(1-x)^{p} d x & =(-1)^{p} \int_{0}^{1} y^{z-p-1}(1-y)^{p} d y \\
& =(-1)^{p} B(z-p, p+1) .
\end{aligned}
$$

Using the well-known equality

$$
B(n, m)=\frac{\Gamma(n) \Gamma(m)}{\Gamma(n+m)},
$$

one can obtain the final result

$$
\begin{aligned}
J_{12} \propto & \frac{1}{z(z-1)}\left\langlen _ { 1 } h _ { c } \left( z-1-n_{2}-n_{2} n_{3}-n_{2} n_{3} n_{4}-\cdots\right.\right. \\
& \left.\left.-n_{2} n_{3} n_{4} \cdots n_{z}\right)\right\rangle .
\end{aligned}
$$

The second type of the jump pairs is considered in a similar way. 
*Corresponding author; telephone: 420266052165, mobile: 420776024705, FAX: 420286581448. Electronic address: taras@fzu.cz

${ }^{1}$ A. A. Chumak and A. A. Tarasenko, Surf. Sci. 91, 694 (1980).

${ }^{2}$ D. A. Reed and G. Ehrlich, Surf. Sci. 102, 558 (1981); 105, 603 (1981).

${ }^{3}$ V. P. Zhdanov, Elementary Physicochemical Processes on Solid Surfaces (Plenum, New York, 1991).

${ }^{4}$ Z. Chvoj, V. Cháb, and H. Conrad, Surf. Sci. 426, 8 (1999).

${ }^{5}$ A. A. Tarasenko and L. Jastrabik, Surf. Sci. 507-510, 108 (2002).

${ }^{6}$ N. A. Tarasenko, A. A. Tarasenko, Z. Bryknar, and L. Jastrabik, Surf. Sci. 562, 22 (2004).

${ }^{7}$ A. A. Tarasenko, L. Jastrabik, and C. Uebing, Phys. Rev. B 57, 10166 (1998).

${ }^{8}$ A. A. Tarasenko, P. M. Tomchuk, and A. A. Chumak, Fluctuations in Bulk and on Surfaces of Solids (Naukova Dumka, Kyiv, Ukraine, 1992), p. 250 (in Russian).

${ }^{9}$ A. A. Tarasenko, L. Jastrabik, F. Nieto, and C. Uebing, Phys. Rev. B 59, 8252 (1999).

${ }^{10}$ A. A. Tarasenko, F. Nieto, L. Jastrabik, and C. Uebing, Phys. Rev. B 64, 075413 (2001).

${ }^{11}$ A. Danani, R. Ferrando, E. Scalas, and M. Torri, Int. J. Mod. Phys. B 11, 2217 (1997).

${ }^{12}$ A. Danani, R. Ferrando, E. Scalas, and M. Torri, Surf. Sci. 402404, 281 (1998).

${ }^{13}$ A. Danani, R. Ferrando, E. Scalas, and M. Torri, Surf. Sci. 409, 117 (1998).

${ }^{14} \mathrm{~J}$. Bardeen and C. Herring, in Imperfections in Nearly Perfect Crystals, edited by W. Shockley (Wiley, New York, 1952), p. 261.
${ }^{15}$ Th. Niemeyer and J. M. J. van Leeuwen, Physica (Amsterdam) 71, 17 (1974).

${ }^{16}$ M. Nauenberg and B. Nienhuis, Phys. Rev. Lett. 33, 1598 (1974).

${ }^{17}$ B. Nienhuis and M. Nauenberg, Phys. Rev. Lett. 35, 477 (1975).

${ }^{18}$ Th. Niemeyer and J. M. J. van Leeuwen, in Phase Transitions and Critical Phenomena, edited by C. Domb and M. S. Green (Academic, New York, 1976), Vol. VI, Chap. 7.

${ }^{19}$ G. D. Mahan and F. H. Claro, Phys. Rev. B 16, 1168 (1977).

${ }^{20}$ K. W. Kehr, R. Kutner, and K. Binder, Phys. Rev. B 23, 4931 (1981).

${ }^{21}$ R. Kutner, K. Binder, and K. W. Kehr, Phys. Rev. B 26, 2967 (1982).

${ }^{22}$ A. Sadiq and K. Binder, Surf. Sci. 128, 350 (1983); 145, 350 (1984).

${ }^{23} \mathrm{~K}$. Kehr and K. Binder, in Applications of the Monte Carlo Method in Statistical Physics, edited by K. Binder, Topics in Current Physics Vol. 36 (Springer Verlag, Berlin, 1987), p. 181.

${ }^{24}$ M. E. J. Newman and G. T. Barkema, Monte Carlo Methods in Statistical Physics (Oxford University Press, Oxford, 2004).

${ }^{25}$ A. B. Bortz, M. H. Kalos, and J. L. Lebovitz, J. Comput. Phys. 17, 10 (1975).

${ }^{26}$ F. M. Bulnes, V. D. Pereyra, and J. L. Riccardo, Phys. Rev. E 58, 86 (1998).

${ }^{27}$ M. A. Novotny, in Annual Reviews of Computational Physics IX, edited by D. Stauffer (World Scientific, Singapore, 2001), p. 153 (also available at http://www.arxiv.org/cond-mat/0109182).

${ }^{28}$ D. A. Reed and G. Ehrlich, Surf. Sci. 102588 (1981).

${ }^{29}$ Z. Alexandrowicz, J. Stat. Phys. 13, 231 (1975); 14, 1 (1976). 